\title{
Rapidly progressive ACTH-dependent Cushing's disease masquerading as ectopic ACTH-producing Cushing's syndrome: illustrative case
}

\author{
Siyuan Yu, BA, ${ }^{1}$ Michael Karsy, MD, PhD, MSc, ${ }^{1}$ Jeffrey Miller, MD, ${ }^{2}$ Stephanie R. Beldick, MSc, ${ }^{3}$ Mark T. Curtis, MD, ${ }^{3}$ \\ Marc Rosen, MD, ${ }^{4}$ and James J. Evans, $\mathrm{MD}^{1,4}$ \\ Departments of ${ }^{1}$ Neurological Surgery, ${ }^{2}$ Endocrinology, ${ }^{3}$ Pathology, and ${ }^{4}$ Otolaryngology, Thomas Jefferson University Hospital, Philadelphia, Pennsylvania
}

\begin{abstract}
BACKGROUND Cushing's disease (CD) remains a challenging condition to diagnose and treat. This case study highlights the challenges of diagnosing CD when faced with discrepant clinical, biochemical, and radiological findings.

OBSERVATIONS A 62-year-old man presented with rapid evolution of symptoms, including depression, fatigue, and extreme muscle atrophy, which resulted in the patient being a wheelchair user over the course of a few months. His rapid clinical course in conjunction with hypercortisolemia in the setting of a pituitary macroadenoma involving the cavernous sinus, two large pulmonary nodules, and urine-free cortisol levels in the thousands suggested an aggressive ectopic adrenocorticotropic hormone (ACTH) source. After extensive testing ruled out CD from an ectopic source and because of the patient's abrupt clinical deterioration, the authors concluded that the source was likely an aggressive pituitary adenoma. Therefore, the authors performed an endonasal transsphenoidal approach for resection of the pituitary adenoma involving the cavernous sinus, and the patient was scheduled for radiosurgery to control tumor progression.
\end{abstract}

LESSONS Although extremely high levels of cortisol and ACTH are associated with ectopic Cushing's syndrome, they may also indicate an aggressive form of $\mathrm{CD}$. Suspicion should be maintained for hypercortisolemia from a pituitary source even when faced with discrepant information that may suggest an ectopic source.

https://thejns.org/doi/abs/10.3171/CASE21151

KEYWORDS Cushing's disease; Cushing's syndrome; hypercortisolism; ectopic adrenocorticotropin

Cushing's syndrome (CS) is characterized by high levels of cortisol in the blood, which may be a result of excessive production of adrenocorticotropic hormone (ACTH) from the pituitary gland or ectopic extrapituitary production. Distinguishing excessive production of cortisol via ACTH-secreting pituitary tumors (ACTH-dependent Cushing's disease [CD]) from ectopic tumors that secrete corticotropin-releasing hormone, ACTH (ectopic Cushing's), or cortisol in an ACTH-independent manner is important to guide treatment. ${ }^{1}$ However, the diagnosis of $A C T H$-dependent $C D$ remains challenging, especially in the setting of unclear imaging and laboratory findings.

Our patient presented with myopathy and difficulty ambulating that rapidly worsened in the span of a year. Magnetic resonance imaging (MRI) revealed a pituitary macroadenoma. However, serological studies, overall clinical deterioration, and suspicious findings on computed tomography (CT) scans of the lungs were all suggestive of an ectopic source, which made the diagnosis challenging.

\section{Illustrative Case}

A 62-year-old man with a past medical history of diabetes, hyperlipidemia, and atrial fibrillation presented with rapidly progressive lower extremity edema, generalized weakness, gait imbalance, and increased frequency of falls over a few months. Chart review revealed episodes of hypokalemia with blood glucose level in the range of $300 \mathrm{mg} / \mathrm{dL}$ and multiple admissions for treatment of hyperglycemia, pneumonia, and metabolic alkalosis. Over a 1-year period, the patient demonstrated increased fatigue, depressive mood, extreme muscle atrophy, and weight

ABBREVIATIONS ACTH = adrenocorticotropic hormone; $C D=$ Cushing's disease; $C S=$ Cushing's syndrome; $C T$ = computed tomography; DST = dexamethasone suppression test; IHC = immunohistochemistry; MRI = magnetic resonance imaging; UFC = urine-free cortisol.

INCLUDE WHEN CITING Published July 5, 2021; DOI: 10.3171/CASE21151.

SUBMITTED March 13, 2021. ACCEPTED April 26, 2021.

(C) 2021 The authors, CC BY-NC-ND 4.0 (http://creativecommons.org/licenses/by-nc-nd/4.0/). 
TABLE 1. Results of $2 \mathrm{mg}$ 48-hour DST and a follow-up $8 \mathrm{mg}$ 48-hour DST

\begin{tabular}{|c|c|c|c|}
\hline Date & $\begin{array}{l}\text { Morning Cortisol } \\
(6.2-19.4 \mu \mathrm{g} / \mathrm{dL})\end{array}$ & $\begin{array}{c}\text { UFC } \\
(5-64 \mu \mathrm{g} / 24 \mathrm{hr})\end{array}$ & $\begin{array}{c}\text { ACTH } \\
(7.2-63 \mathrm{pg} / \mathrm{mL})\end{array}$ \\
\hline \multicolumn{4}{|c|}{2 mg 48-hr DST* } \\
\hline $3 / 2020$ & 11.1 & & 135.8 \\
\hline $3 / 2020$ & & 25 & \\
\hline $3 / 2020$ & 17.1 & & 162.3 \\
\hline $3 / 2020$ & 25 & 215 & 201.9 \\
\hline \multicolumn{4}{|c|}{8 mg 48-hr DST* } \\
\hline $4 / 2020$ & 14.9 & & 163 \\
\hline $4 / 2020$ & & 74 & \\
\hline $4 / 2020$ & 26.2 & & \\
\hline $4 / 2020$ & 71.8 & 1,656 & 583 \\
\hline
\end{tabular}

*Dexamethasone levels were not available for these tests.

loss of approximatly $60 \mathrm{lb}$, resulting in the patient becoming a wheelchair user. The patient did not demonstrate classic cushingoid features.

Blood test results demonstrated a morning cortisol level of $72 \mu \mathrm{g} /$ $\mathrm{dL}$ (normal range 6.2-19.4 $\mu \mathrm{g} / \mathrm{dL}$ ); however, subsequent 24-hour urine-free cortisol (UFC) levels and $1 \mathrm{mg}$ dexamethasone suppression test (DST) were normal despite two separate measurements. Other pituitary hormone levels were unremarkable. A 48-hour $2 \mathrm{mg}$ DST and $8 \mathrm{mg}$ high-dose DST (Table 1) demonstrated elevated ACTH levels and a failure of cortisol suppression. Several additional 24-hour free cortisol levels were attempted, some in combination with DSTs, with four measurements showing elevated values. However, UFC levels of 1,656 $\mu \mathrm{g} / 24$ hours (normal level 5-64 $\mu \mathrm{g} / 24$ hours) suggested an ectopic cortisol source (Table 2). A chest and abdomen CT revealed two lung cysts (Fig. 1), one of which was biopsied and was unremarkable for a lung tumor. Additionally, an autoimmune panel and a muscle biopsy were both nonrevealing. MRI showed a $1.3-\mathrm{cm}$ pituitary macroadenoma involving the left cavernous sinus (Fig. 1).

Due to the patient's progressive failure to thrive and lack of definitive ectopic ACTH source, he underwent an endoscopic resection of the pituitary macroadenoma. Intraoperatively, gross total resection of the tumor involving the sella, clivus, and left cavernous sinus was achieved. Pathology revealed corticotropic pituitary adenoma staining strongly for ACTH and elevated Ki- 67 proliferation index of $17 \%$ using immunohistochemistry ( $\mathrm{HC}$ ) staining (Fig. 2). Postoperative day 1 morning cortisol revealed a level of $30.7 \mu \mathrm{g} / \mathrm{dL}$, which progressively decreased to a cortisol level of $5.6 \mu \mathrm{g} / \mathrm{dL}$ at discharge on postoperative day 5 . The patient was subsequently placed on steroid replacement. At 3 months follow-up, with the patient on prednisone $7.5 \mathrm{mg}$ daily, blood test results revealed an ACTH of $43 \mathrm{pg} / \mathrm{mL}$ and a morning cortisol level of $3 \mu \mathrm{g} / \mathrm{dL}$. He regained $15 \mathrm{lb}$ and was able to ambulate independently with a cane, and his leg swelling had decreased significantly. However, 8 months after surgery, the patient's cortisol levels started to climb, which was indictive of recurrence (Table 3). Due to symptomatic refractory hypercortisolemia, the patient was started on ketoconazole, and underwent stereotactic radiosurgery. As his cortisol and ACTH levels continued to be elevated, he underwent bilateral adrenalectomies. He only then began showing improved cortisol levels, mentation, and functional status.

\section{Discussion \\ Observations}

This report presents the case of a 62-year-old man with rapid clinical deterioration secondary to hypercortisolemia. In the setting of unclear localization, including a pituitary macroadenoma concerning for hypercortisolemia of pituitary origin versus pulmonary nodules, UFC levels in the thousands, and the aggressive nature of the clinical course concerning for hypercortisolemia of ectopic origin, the diagnosis was delayed. After extensive testing ruling out an ectopic source, the patient ultimately underwent an endonasal transsphenoidal approach for resection of the pituitary adenoma involving the cavernous sinus that resulted initial in biochemical remission of the patient's $C D$. Due to refractory hypercortisolemia, the patient required radiosurgery, ketoconazole treatment, and eventually bilateral adrenalectomies.

\section{Lessons}

CD accounts for $70 \%$ to $80 \%$ of ACTH-dependent CS, with a prevalence of 40:1,000,000 and occurrence more common in women, with a 3:1 female to male ratio. ${ }^{1}$ The clinical features of $C D$ are highly variable and may include obesity, abdominal striae, osteoporosis, delayed wound healing, moon face, and dorsal cervical fat pad, and it may take as long as 2 to 4 years after initial symptom onset to diagnosis. A prolonged and persistent state of hypercortisolemia increases a patient's risk of metabolic syndrome, hyperglycemia, opportunistic infections, neuropsychiatric disorders, decreased quality of life, and cardiovascular complications. The mortality rate with $C D$ can be as

TABLE 2. 24-hour UFC test results before surgical treatment

\begin{tabular}{lccr}
\hline UFC Date* & UFC Per Volume/Total Volume & 24-hr UFC $(5-64 \mu \mathrm{g} / 24 \mathrm{hr})$ & Test Performed \\
\hline $3 / 2020$ & Outside lab testing & $27 \mu \mathrm{g} / 24 \mathrm{hr}$ & Screening test \\
\hline $3 / 2020$ & Unable to void & - & Screening test \\
\hline $3 / 2020$ & $18 / 2,500 \mathrm{~mL}$ & $25 \mu \mathrm{g} / 24 \mathrm{hr}$ & $2 \mathrm{mg} 48-\mathrm{hr}$ DST \\
\hline $3 / 2020$ & $78 / 2,750 \mathrm{~mL}$ & $215 \mu \mathrm{g} / 24 \mathrm{hr}$ & $2 \mathrm{mg} \mathrm{48-hr} \mathrm{DST}$ \\
\hline $4 / 2020$ & $32 / 2,300 \mathrm{~mL}$ & $74 \mu \mathrm{g} / 24 \mathrm{hr}$ & $8 \mathrm{mg} \mathrm{48-hr} \mathrm{DST}$ \\
\hline $4 / 2020$ & $828 / 2,000 \mathrm{~mL}$ & $1,656 \mu \mathrm{g} / 24 \mathrm{hr}$ & $8 \mathrm{mg} \mathrm{48-hr} \mathrm{DST}$ \\
\hline $5 / 2020$ & $188 / 2,000 \mathrm{~mL}$ & $376 \mu \mathrm{g} / 24 \mathrm{hr}$ & $8 \mathrm{mg} 48-\mathrm{hr}$ DST \\
\hline $5 / 2020$ & $1,410 / 2,750 \mathrm{~mL}$ & $3,878 \mu \mathrm{g} / 24 \mathrm{hr}$ & $8 \mathrm{mg} \mathrm{48-hr} \mathrm{DST}$ \\
\hline
\end{tabular}

\footnotetext{
*Patient had a normal creatine level $1.1(0.76-1.27 \mathrm{mg} / \mathrm{dL})$ in January 2020.
} 


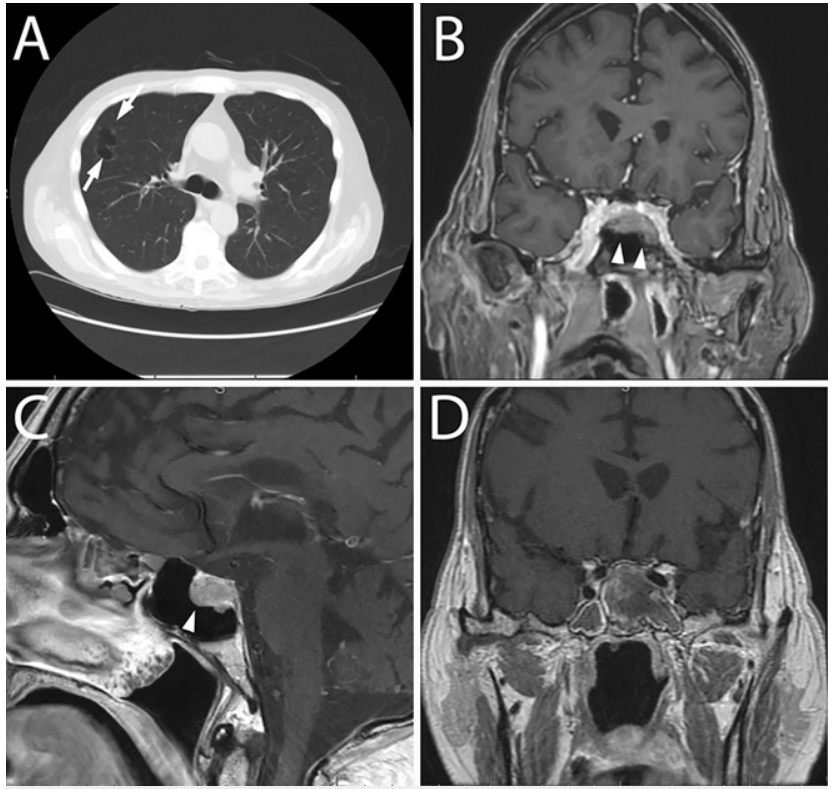

FIG. 1. Preoperative and postoperative MRI of an aggressive ACTHsecreting pituitary adenoma. A: Preoperative workup, including CT of the chest, demonstrated two cystic pulmonary lesions (arrows) that were biopsied to rule out an ectopic source of ACTH secretion. Preoperative coronal (B) and sagittal (C) postcontrast T1 images demonstrate a hypointense pituitary macroadenoma (arrowheads) with extension into the left cavernous sinus. D: Postoperative T1 coronal image with contrast showing removal of the lesion and expected postoperative changes in the sphenoid sinus and cavernous sinus enhancement.

much as four times higher than that of the general population, with cardiovascular complications being the leading cause of death. ${ }^{1}$

The initial workup of CD includes physical examination, evaluation of exogenous glucocorticoid administration, 24-hour UFC, or three midnight salivary cortisol tests. ${ }^{2-4}$ Testing should be performed on separate occasions. Overall sensitivity for these tests ranges from $80 \%$ to $98 \%$, with important caveats for specific tests. ${ }^{4}$ Once CD is suspected, confirmatory testing can be performed that involves high-dose DST (8 mg), measurement of ACTH levels, and brain MRI to localize the pituitary lesion. CD demonstrates cortisol levels that are 1 to 2 times normal levels as well as ACTH levels that are 3 times normal levels, whereas CS can have cortisol levels that are 3 to 4 times normal and ACTH levels that are at or above 10 to 100 times normal. If CS is suspected, wholebody imaging with CT of the chest and abdomen should be conducted to look for an ectopic source. Additional identification of ectopic CS may involve the use of gallium-68 dotatate positron emission tomography, which may be helpful in identifying nonpituitary sources of ACTH in neuroendocrine tumors. ${ }^{5-7}$ Inferior petrosal sinus sampling may be attempted to confirm a diagnosis of $C D$ or evaluate for laterality of the tumor within the sella in cases that are nonlesional on MRI. Guidelines from the Endocrine Society and the European Society of Endocrinology help clarify testing but do not completely agree on workup and testing cutoff values. $^{5-7}$

Diagnosis of CD may be challenging when patients do not exhibit concordant clinical signs, biochemical testing, and imaging. One series looked at biochemical profiles of ectopic CS and CD. It found that ectopic CS had much higher ACTH levels and that ACTH levels
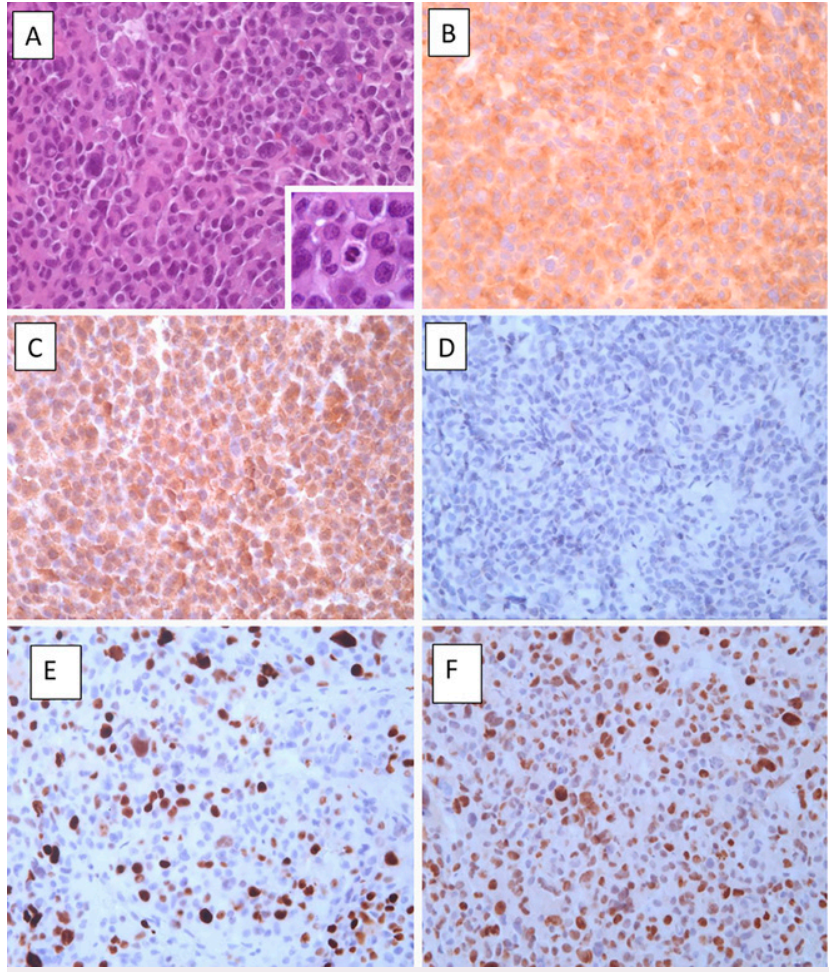

FIG. 2. Histology of pituitary tumor with hematoxylin and eosin and $\mathrm{IHC}$ staining demonstrating an aggressive $\mathrm{ACTH}$-secreting tumor by elevated Ki-67 index. A: Hematoxylin and eosin stain of the pituitary adenoma comprised of sheets of cells with eosinophilic cytoplasm and mildly pleomorphic nuclei (original magnification $\times 600$ ). Rare mitotic figures were present (inset, original magnification $\times 1,000$ ). $\mathbf{B}$ : $I \mathrm{HC}$ stain for chromogranin, a marker of neuroendocrine cells, including those found in pituitary adenomas, shows strong staining of this pituitary adenoma (original magnification $\times 600$ ). C: $I H C$ shows strong, diffuse staining for ACTH in the pituitary adenoma cells, demonstrating ACTH production by the adenoma cells. This strong pattern of staining is compatible with this adenoma being an ACTH-secreting pituitary adenoma (original magnification $\times 600$ ). $\mathrm{D}: \mathrm{IHC}$ is negative for thyroid transcription factor 1 (TTF-1). TTF-1 is a nuclear marker present in cells of lung, thyroid, and neurohypophyseal origin and in peripheral systemic neuroendocrine tumors. Given the concerning findings on chest CT (see Fig. 1), the absence of staining for TTF-1 supports the diagnosis of pituitary adenoma rather than a neuroendocrine tumor of systemic origin (original magnification $\times 400$ ). E: Positive $\mathrm{IHC}$ staining for Ki-67, a nuclear marker of cell cycle activity. The Ki-67 proliferation index is $17 \%$, which is elevated and may indicate more aggressive tumor behavior (original magnification $\times 400$ ). $\mathrm{F}$ : $\mathrm{HC}$ is positive for nuclear staining of $p 53$, an atypical finding in pituitary adenomas. The role of p53 expression in prognostication of pituitary adenomas is unclear (original magnification $\times 400$ ).

$>200 \mathrm{pg} / \mathrm{mL}$ could indicate an ectopic source. ${ }^{8}$ The variation in cutoff for $C D$ diagnosis also may result in alteration of test sensitivity/specificity. The Japanese Ministry of Health diagnostic criteria for CD indicates use of low-dose $(0.5 \mathrm{mg})$ DST, with $5.0 \mu \mathrm{g} / \mathrm{dL}$ as the cutoff value. This combination showed a sensitivity of $97 \%$ and a specificity of $88 \%$ compared to the standard $1 \mathrm{mg}$ DST, which showed a sensitivity of $75 \%$ and specificity of $87 \%$ for initial screening test currently recommended by endocrinology guidelines when studied in the Japanese population. ${ }^{9}$ Use of circadian variations by measurement of 
TABLE 3. Laboratory values indicting a recurrence of patient's CD

\begin{tabular}{lcc}
\hline Date & Test & Results \\
\hline $11 / 2020$ & ACTH & $44.6(7.2-63.3 \mathrm{pg} / \mathrm{mL})$ \\
\hline $11 / 2020$ & Morning cortisol & $3(6.2-19.4 \mathrm{pg} / \mathrm{mL})$ \\
\hline $2 / 2021$ & ACTH & $159.0(7.2-63.3 \mathrm{pg} / \mathrm{mL})$ \\
\hline $2 / 2021$ & Morning cortisol & $14.1(6.2-19.4 \mathrm{pg} / \mathrm{mL})$ \\
\hline $2 / 2021$ & UFC & $323(5-64 \mu \mathrm{g} / 24 \mathrm{hr})^{*}$ \\
\hline $2 / 2021$ & ACTH & $176(7.2-63.3 \mathrm{pg} / \mathrm{mL})$ \\
\hline $3 / 2021$ & Morning cortisol & $83.2(6.2-19.4 \mathrm{pg} / \mathrm{mL})$ \\
\hline $3 / 2021$ & UFC & No result \\
\hline $3 / 2021$ & ACTH & $228.0(7.2-63.3 \mathrm{pg} / \mathrm{mL})$ \\
\hline
\end{tabular}

*02/2021 creatinine level $1.5(0.76-1.27 \mathrm{mg} / \mathrm{dL})$.

cortisol and ACTH pulsatility without concurrent use of dexamethasone suppression testing also showed high sensitivity and specificity for distinguishing $C D$ from a pseudo-Cushing's state..$^{10}$ Separating $C D$ from an ectopic source seems straightforward but can be difficult from a biochemical perspective. High-dose DST gives a variable sensitivity and specificity of $60 \%$ to $100 \%$ and $65 \%$ to $100 \%$ for $C D$, respectively. A reported series of ectopic tumors revealed that when a cutoff of $60 \%$ suppression was used for high-dose DST, the sensitivity for diagnosing ectopic cortisol sources increased from $91 \%$ to $97 \% .{ }^{11}$ Caveats include mention that ectopic tumors are appropriately suppressed by high-dose DST $5 \%$ to $25 \%$ of the time, and up to $20 \%$ of patients with CD fail to suppress with high-dose DST. ${ }^{12}$ Some authors have argued that high-dose DST cannot accurately distinguish between well-differentiated systemic tumors and pituitary adenomas. ${ }^{13}$

Use of alternative tests in addition to standard tests may be helpful in guiding $C D$ diagnosis but remains limited. One alternative test includes use of desmopressin (DDAVP). DDAVP is structurally similarly to vasopressin, which acts on the kidneys to mediate water reabsorption. It also stimulates release of ACTH and cortisol in a proportion of patients with ACTH-mediated CS while having minimal effect on healthy people. One study showed $90.8 \%$ sensitivity and $94.6 \%$ specificity in diagnosing $C D$ in a population of patients suspected of having $\mathrm{CS} .^{14}$ Additional application of DDAVP response may be used to monitor recurrence in CD. Loss of DDAVP response after surgery is correlated with favorable outcome whereas return of response corresponds with recurrence. ${ }^{15}$

Our case involving $C D$ is extremely unusual given the high cortisol and ACTH levels and the rapid deterioration of the patient's clinical symptoms that are highly suggestive of $C D$ from an ectopic source. $\mathrm{IHC}$ revealed positive staining for $\mathrm{ACTH}$ pituitary adenoma with an elevated Ki-67 proliferation index of $17 \%$. Resolution of cortisol and ACTH levels, along with clinical improvement, confirmed the pituitary source of the disease. It is unclear whether the high Ki-67 index in this patient may have resulted in a more aggressively growing tumor, which could have impacted the high cortisol and ACTH levels as well as the rapid clinical progression of symptoms. Our case serves as a reminder that the workup and diagnosis of $C D$ may be challenging given the incongruous biochemical, clinical, and radiological information. Although our dedicated pituitary team has more than 20 years of experience in managing patients with $\mathrm{CD}$, the diagnosis in our patient was ultimately made only after extensive testing ruled out ectopic CS, which resulted in delayed surgical management and worsening of our patient's clinical symptoms. The case further suggests that a high level of suspicion should be maintained for hypercortisolemia from a pituitary source even when faced with unclear clinical information that might suggest an ectopic source. Improved diagnostic criteria and interdisciplinary care among neurosurgery and endocrinology can help with proper patient selection and earlier treatment.

\section{Acknowledgments}

Publication of this study was made possible in part by support from the Thomas Jefferson University Open Access Fund.

\section{References}

1. Nishioka H, Yamada S. Cushing's disease. J Clin Med. 2019;8(11):1951.

2. Daniel E, Newell-Price JD. Diagnosis of Cushing's disease. Pituitary. 2015;18(2):206-210.

3. Nieman LK, Biller BMK, Findling JW, et al. Treatment of Cushing's syndrome: an Endocrine Society clinical practice guideline. J Clin Endocrinol Metab. 2015;100(8):2807-2831.

4. Newell-Price JDC. Cushing disease. In: Melmed S, ed. The Pituitary. 4th ed. Academic Press; 2017:515-571.

5. Papadakis GZ, Bagci U, Sadowski SM, et al. Ectopic ACTH and CRH co-secreting tumor localized by 68Ga-DOTA-TATE PET/CT. Clin Nucl Med. 2015;40(7):576-578.

6. Diwaker C, Shah RK, Patil V, et al. 68Ga-DOTATATE PET/CT of ectopic Cushing syndrome due to appendicular carcinoid. Clin NuCl Med. 2019;44(11):881-882.

7. Wannachalee T, Turcu AF, Bancos I, et al. The clinical impact of $\left[{ }^{68} \mathrm{Ga}\right]$-DOTATATE PET/CT for the diagnosis and management of ectopic adrenocorticotropic hormone-secreting tumours. Clin Endocrinol (Oxf). 2019;91(2):288-294.

8. Sathyakumar S, Paul TV, Asha HS, et al. Ectopic Cushing syndrome: a 10-year experience from a tertiary care center in Southern India. Endocr Pract. 2017;23(8):907-914.

9. Kageyama K, Oki Y, Sakihara S, et al. Evaluation of the diagnostic criteria for Cushing's disease in Japan. Endocr J. 2013;60(2): 127-135.

10. Cunningham JM, Buxton OM, Weiss RE. Circadian variation in Cushing's disease and pseudo-Cushing states by analysis of $F$ and ACTH pulsatility. J Endocrinol Invest. 2002;25(9):791-799.

11. Isidori AM, Kaltsas GA, Mohammed S, et al. Discriminatory value of the low-dose dexamethasone suppression test in establishing the diagnosis and differential diagnosis of Cushing's syndrome. J Clin Endocrinol Metab. 2003;88(11):5299-5306.

12. Hayes AR, Grossman AB. The ectopic adrenocorticotropic hormone syndrome: rarely easy, always challenging. Endocrinol Metab Clin North Am. 2018;47(2):409-425.

13. Young $J$, Haissaguerre M, Viera-Pinto $O$, et al. Management of endocrine disease: Cushing's syndrome due to ectopic ACTH secretion. An expert operational opinion. Eur J Endocrinol. 2020;182(4):R29-R58.

14. Rollin GA, Costenaro F, Gerchman F, et al. Evaluation of the DDAVP test in the diagnosis of Cushing's disease. Clin Endocrinol (Oxf). 2015;82(6):793-800.

15. Vassiliadi DA, Tsagarakis S. Diagnosis of endocrine disease: the role of the desmopressin test in the diagnosis and follow-up of Cushing's syndrome. Eur J Endocrinol. 2018;178(5):R201-R214.

\section{Disclosures}

Dr. Karsy reported other from Thieme Medical Publishing and Cyrus Surgical outside the submitted work. Dr. Evans reported other from Mizuho outside the submitted work. 


\section{Author Contributions}

Conception and design: Evans, Yu, Karsy, Miller, Curtis, Rosen. Acquisition

of data: Yu, Miller, Beldick, Curtis. Analysis and interpretation of data: Evans,

Yu, Karsy, Miller, Beldick, Curtis. Drafting the article: Evans, Yu, Karsy, Miller,

Beldick, Curtis. Critically revising the article: Evans, Karsy, Miller, Rosen.

Reviewed submitted version of manuscript: Evans, Karsy, Miller, Beldick,

Rosen. Approved the final version of the manuscript on behalf of all authors:

Evans. Statistical analysis: Evans, Miller. Administrative/technical/material

support: Miller. Study supervision: Evans, Miller.

\section{Correspondence}

James J. Evans: Thomas Jefferson University Hospital, Philadelphia, PA. james.evans@jefferson.edu. 